
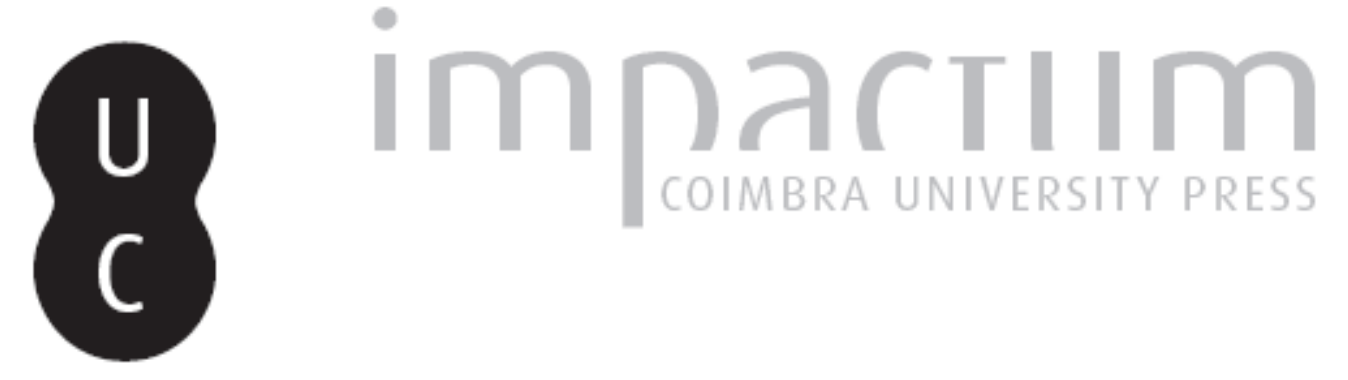

\title{
La epifanía de Dioniso: una interpretación del Fragmento 357 PMG de Anacreonte a partir de la consideración de sus ediciones modernas
}

Autor(es): Larrosa, Marina

Publicado por: Centro de Literatura Portuguesa; Imprensa da Universidade de Coimbra

URL

persistente:

URI:http://hdl.handle.net/10316.2/37789

DOI:

DOI:http://dx.doi.org/10.14195/2182-8830_3-1_8

Accessed : $\quad$ 26-Apr-2023 10:51:45

A navegação consulta e descarregamento dos títulos inseridos nas Bibliotecas Digitais UC Digitalis, UC Pombalina e UC Impactum, pressupõem a aceitação plena e sem reservas dos Termos e Condições de Uso destas Bibliotecas Digitais, disponíveis em https://digitalis.uc.pt/pt-pt/termos.

Conforme exposto nos referidos Termos e Condições de Uso, o descarregamento de títulos de acesso restrito requer uma licença válida de autorização devendo o utilizador aceder ao(s) documento(s) a partir de um endereço de IP da instituição detentora da supramencionada licença.

Ao utilizador é apenas permitido o descarregamento para uso pessoal, pelo que o emprego do(s) título(s) descarregado(s) para outro fim, designadamente comercial, carece de autorização do respetivo autor ou editor da obra.

Na medida em que todas as obras da UC Digitalis se encontram protegidas pelo Código do Direito de Autor e Direitos Conexos e demais legislação aplicável, toda a cópia, parcial ou total, deste documento, nos casos em que é legalmente admitida, deverá conter ou fazer-se acompanhar por este aviso.

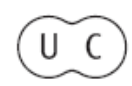




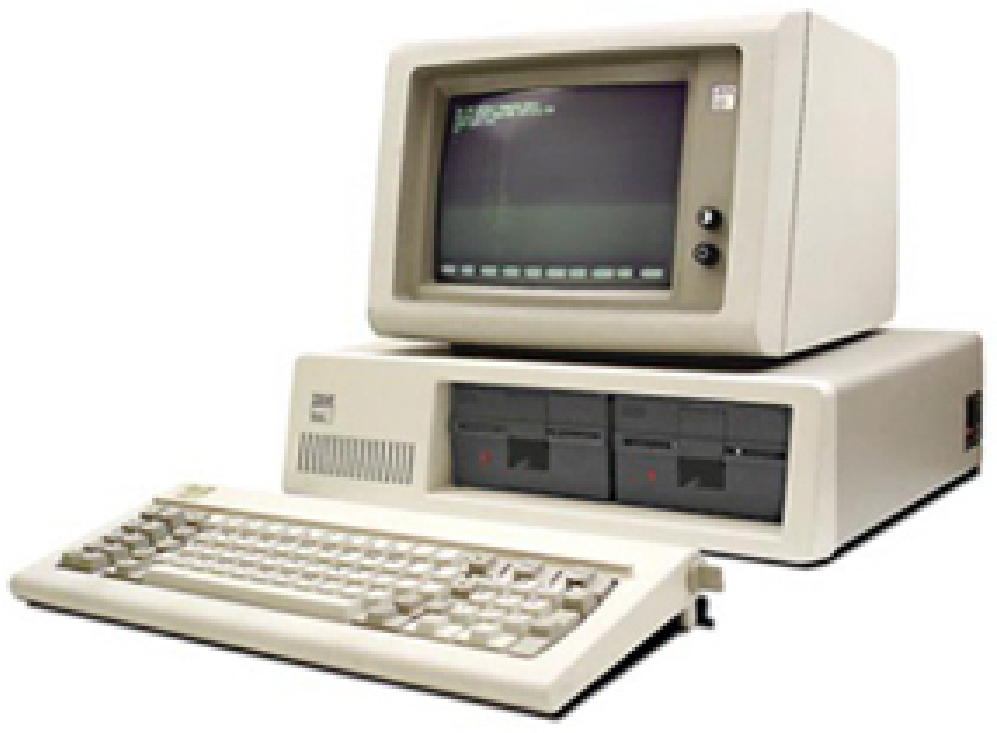

Vol. 3.1 (2015)

ISSN 2182-8830

'Artes, Média e Cultura Digital'

Paulo Silva Pereira e Pedro Serra (orgs.) 


\title{
La Epifanía de Dioniso: Una Interpretación del Fragmento 357 PMG de Anacreonte a partir de la Consideración de sus Ediciones Modernas MARINA LARROSA \\ Universidad Nacional de Rosario (UNR) | CONICET
}

\begin{abstract}
Resumen
En el presente artículo realizamos una interpretación de un famoso poema del poeta griego Anacreonte (fr. 357 PMG) teniendo en cuenta su peculiar historia textual. Planteamos que el poema, una plegaria al dios Dioniso, presenta rasgos que lo aproximan al género del enigma. Pero más allá de esta hipótesis, nos interesa ofrecer esta lectura como un ejemplo de cómo el conocimiento de las distintas ediciones de un texto puede arrojar luz sobre algunos aspectos poéticos que, caso contrario, podrían permanecer velados, y cómo puede asimismo estimular la creatividad en la propia tarea de interpretación. Palabras clave: edición; filología; Anacreonte; enigma; interpretación.
\end{abstract}

\section{Abstract}

In this article we offer an interpretation of a well known poem by the ancient Greek poet Anacreon (fr. $357 P M G$ ) by paying attention to its peculiar textual history. We suggest that the poem - a prayer to Dionysus - presents some of the features that are characteristic of the riddle. But first and foremost we are interested in offering this interpretation as an example of how knowing and working with the different editions of a single text can throw light on certain poetic aspects that would otherwise remain veiled, and how it can also stimulate the creative activity of the interpreter herself. Keywords: edition; philology; Anacreon; riddle; interpretation.

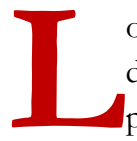
os fragmentos líricos de Anacreonte atestiguan una relación inusitada entre un poeta y el terrible Eros. El viejo poeta de Teos parece no poder (y no querer) desasirse del dominio del joven dios, a quien responsabiliza por su inclinación al fácil enamoramiento (cf. frs. 358, 400, $413 P M G$ ) y de quien suele quejarse cuando fracasa en sus conquistas (frs. 378, 379 PMG). Sin embargo, cuando decide dirigirse a una divinidad para que lo auxilie en una ardua empresa erótica, es Dioniso quien aparece como epikouros. Claramente, Eros es, al igual que Hades, demasiado cruel como para recibir súplicas humanas.

Proponemos, así, introducirnos en nuestra interpretación de la famosa plegaria a Dioniso, un breve poema de Anacreonte de Teos identificado 
como el fragmento 357 en la edición de poetas líricos de Page (1962). ${ }^{1}$ Nuestro objetivo, empero, no será simplemente el comentario literario del poema, sino que ante todo nos interesa ofrecer una lectura sugerida por su particular historia textual. Con ello pretendemos ilustrar cómo el conocimiento de las ediciones de un texto puede ayudar a su interpretación. El trabajo constará de dos partes. Primero realizaremos un recorrido por algunas ediciones del poema de Anacreonte y mostraremos las dificultades textuales a las que debieron enfrentarse los editores. Dicho recorrido nos llevará a plantear una hipótesis de lectura, a saber: que la tardía recuperación del texto, no leído por varios siglos como una plegaria a Dioniso sino a Eros, permite detectar una dificultad en relación con la identidad del dios, lo que nos lleva a pensar en una búsqueda intencionada del poeta de ocultar dicha identidad para que el efecto final sea el de una auténtica revelación. Se podría decir, así, que el poema presentaría una estructura quasi enigmática. Esto será analizado en la segunda parte del trabajo.

A continuación reproducimos el texto con su respectivo aparato crítico según la edición de Page (1962: 182), acompañado de una traducción propia, cuyo objetivo no es otro que el de ayudar en la lectura del original griego.

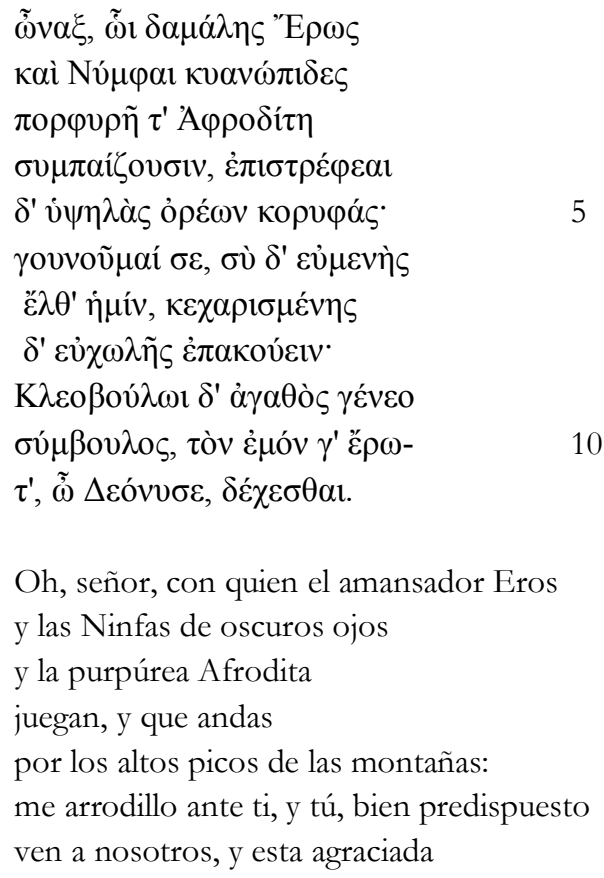

${ }^{1}$ Nos apoyamos en Gentili (1958: 13), quien a su vez sigue a Bergk, a Wilamowitz, a Münsher y a Meyer, y en Del Grande (1959: 203) para nuestro abordaje de la plegaria de Anacreonte como un poema que nos habría sido transmitido de forma completa. Reconocemos, empero, la imposibilidad de comprobar su integridad. Agradezco a los dos evaluadores anónimos por sus útiles sugerencias para enriquecer el contenido del presente artículo. 
plegaria escucha:

para Cleóbulo vuélvete un buen

consejero, para que mi amor,

oh, Dioniso, acepte.

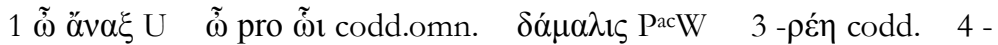

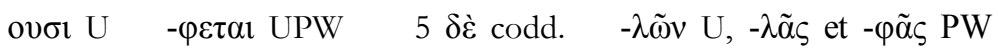

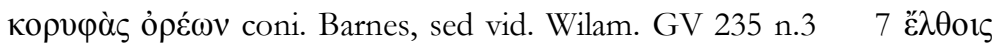

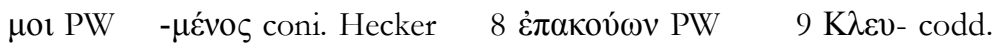

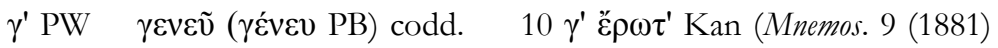

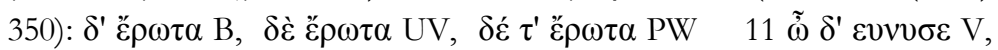
$\tilde{\omega} \delta^{\prime} \varepsilon v \tilde{v} v \ddot{v} \sigma \varepsilon \mathrm{P}, \tilde{\omega} \delta^{\prime} \varepsilon \tilde{v} v \tilde{v} v \sigma \varepsilon \mathrm{UB}\left(\dot{\omega} 1 \delta^{\prime} \mathrm{U}\right)$, corr. Emperius, L. Dindorf $\left(\Delta \varepsilon v^{-}\right) \quad$ fort. $\left(\delta^{\prime} \varepsilon \dot{\varepsilon} \rho \omega \tau^{\prime}\right) \ldots \delta \varepsilon \chi \varepsilon \dot{\varepsilon} \sigma \theta \omega$

\section{La epifanía textual de Dioniso}

La plegaria a Dioniso de Anacreonte nos es transmitida por Dion Crisóstomo en su segundo discurso sobre la monarquía. Allí Dion nos presenta una conversación entre Alejandro y su padre Filipo II de Macedonia, en la que el primero defiende su preferencia por la poesía homérica, a la que considera edificante para todo ser humano y especialmente apropiada a la educación de un rey. Dentro de este contexto el poema de Anacreonte es citado como ejemplo de una plegaria indigna de un monarca. En tanto se trata de la única fuente, las variantes textuales del poema de Anacreonte corresponden a los distintos manuscritos de la obra de Dion, a los que podemos sumar algunas referencias circunstanciales al texto anacreóntico. ${ }^{2} \mathrm{El}$ códice más antiguo, el Vaticanus Graecus 99 (V), data del siglo XI, al igual que el siguiente, el Urbinas Graecus 124 (U). El de datación más moderna es el Meermannianus 67 (M), del siglo XVI. Si observamos el aparato crítico del poema, advertiremos que en el verso undécimo la palabra $\Delta \varepsilon$ óvvбє es una corrección de Adolphus Emperius, autor de una edición decimonónica de la obra de Dion. En los códices, según nos indica Page, el texto va variando, cada vez con segmentaciones más pronunciadas. V presenta $\tilde{\omega} \delta^{\prime} \varepsilon v v v \sigma \varepsilon$, mientras que U ofrece la lectura $\dot{\omega} 1 \delta^{\prime} \varepsilon \tilde{v}$ vข̃v $\sigma \varepsilon$. En el Parisinus 2958 (B), bastante más tardío que U, pero de la misma familia, se corrige la primera palabra y se la reemplaza por $\tilde{\omega} \delta$ '. Finalmente, en $\mathrm{P}$ (Palatinus 117), del siglo XV, parece haberse aceptado la corrección de B,

\footnotetext{
${ }^{2}$ Herodiano (I 79.13 en la edición de Lentz) y Hesiquio $(s . \nu . \delta \alpha \mu \alpha ́ \lambda \eta v)$ se refieren al

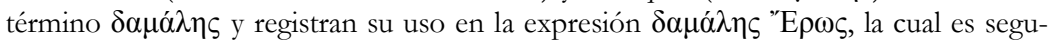
ramente cita del primer verso de nuestro poema. Una tercera referencia la encontramos en Claudio Eliano (De natura animalium 4.2), quien menciona la singularidad de una paloma color púrpura ( $\pi$ о $\varphi \varphi \rho \tilde{v} v$ ), a la que identifica con Afrodita, de acuerdo con la descripción que hiciera Anacreonte de la diosa.
} 
aunque con la eliminación de la $\vee$ final del adverbio: $\tilde{\omega} \delta^{\prime} \varepsilon \tilde{v}$ vü $\sigma \varepsilon$. Se observa claramente cómo el texto se va alejando cada vez más de la palabra, creemos, original, pues el códice más antiguo es el que menos ha segmentado el nombre de Dioniso y, de hecho, en una lectura en voz alta este se mantiene intacto. Pero claramente $\varepsilon v v v \sigma \varepsilon$ no significa nada y de allí parecen surgir las distintas variaciones y segmentaciones que intentan ofrecerle al verso algún sentido.

También el primer verso del himno ha presentado sus propios problemas textuales. Page nos informa que los códices transmiten en su totalidad la interjección $\tilde{\tilde{\omega}}$ en lugar del pronombre relativo en dativo, lo que convierte al dios inmediatamente nombrado (sc. Eros) en el anax a quien se destina la plegaria. Theodor Bergk, el primer editor de Anacreonte que separó los fragmentos genuinos de las canciones del corpus apócrifo hallado por Estienne, y también el primero luego de la corrección al verso undécimo que hiciera Emperius, resume de la siguiente manera la suerte del poema de Anacreonte:

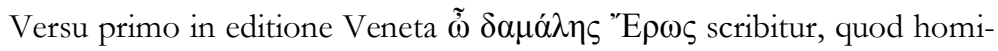
nes doctos in errorem induxit, ut crederent carmen venustissimum in Amorem scriptum esse. Solus Emperius peripexit, poetam invocare Bacchi (...). (Bergk, 1834: 77)

Observemos a continuación algunas soluciones editoriales, a modo de ilustrar las dificultades que presentó la lectura del poema.

La editio princeps de la obra de Anacreonte es de 1554 y fue llevada a cabo por Henri Estienne (Henricus Stephanus). En realidad, lo que motivó esta primera edición fue el hallazgo que el propio Estienne hiciera del corpus pseudo-anacreóntico, al cual estimó auténtico en cuanto a la atribución autoral. Hasta ese momento se conocían solamente algunos fragmentos de Anacreonte por transmisión indirecta, como el caso de nuestra plegaria. Luego de dicho descubrimiento, el humanista francés decidió dar a conocer los nuevos poemas anacreónticos, a los que sumó algunos fragmentos ya conocidos en un apéndice, acompañados estos de algunos textos de Alceo y dos poemas de Safo. Sin embargo, la plegaria no fue incluida en esta primera edición de Anacreonte, sino que aparece en una edición, también de Estienne, de poetas líricos griegos, publicada en 1560. Reproducimos aquí el texto en prosa que se ofrece en la edición mencionada: ${ }^{3}$

${ }^{3}$ Lamentablemente no hemos podido consultar la editio princeps de Anacreonte, pero Michelangeli (1882: 302) nos ratifica la ausencia de la plegaria a Dioniso en ella y, en cambio, su reproducción en la mencionada edición de los líricos. Debemos señalar, empero, que Estienne realiza dos ediciones de los líricos: de la primera, de 1560, hemos obtenido el texto de Anacreonte que reproducimos; la segunda, del año 1566, presenta, sorprendentemente, una versión del texto con la lección $\tilde{\omega}$ en lugar de $\tilde{\omega}$ en lo que sería el primer verso. Claramente, esta segunda fue desechada por los sucesores 


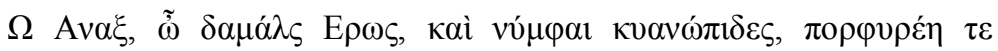

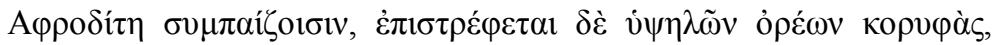

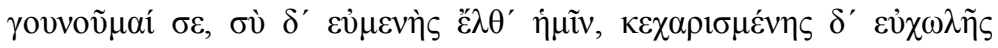

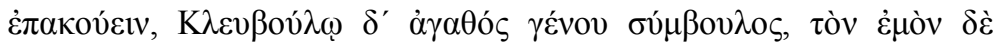

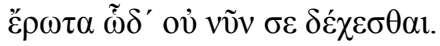

De las ediciones de Anacreonte posteriores a la de Estienne, es la de Longepierre (1692) la primera que incluye este fragmento. En ella Longepierre mantiene el texto tal cual lo presentara su compatriota, pero anota algunas sugerencias. Así, con respecto a los dos primeros versos $(\Omega$ Av $\alpha \xi \tilde{\omega}$

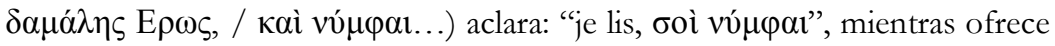

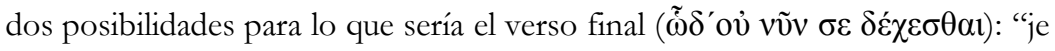

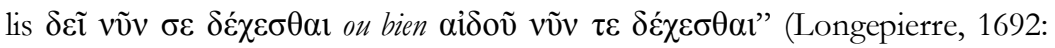
326). La propuesta textual de Longepierre en el primer verso es interesante, porque resuelve con el pronombre de segunda persona en dativo el régimen de la preposición oúv, unida al verbo. Es cierto que la preposición, en lugar de requerir un complemento, podría tener un sentido adverbial ("entre ellos"), exigiendo para este caso únicamente un sujeto en plural. ${ }^{4}$ Pero si el texto de Estienne pide ser comprendido de este último modo, presentando un sujeto que incluiría implícitamente tanto a las Ninfas y a Afrodita como a Eros, no puede, sin embargo, resolver la falsa concordancia entre el verbo en tercera persona del plural y el sujeto gramatical, pues este sería, en realidad, una segunda persona del plural. Longepierre, en cambio, resuelve este problema porque con el pronombre en dativo deja a Eros fuera del sujeto del verbo $\sigma v \mu \pi \alpha i \zeta o v \sigma l(v)$. Con el cambio que propone, el poema habría comenzado: "Oh, señor, oh, amansador Eros, contigo las ninfas de oscuros ojos y la purpúrea Afrodita juegan”, con lo cual se acerca en algún punto al texto actual. Sin embargo, en relación con sus propuestas para el verso final, Longepierre se debe alejar demasiado de la lectura de los códices. Dejando a un lado las incompatibilidades métricas (un factor de cuya consideración una versión en prosa puede muy oportunamente prescindir), la transformación de $\tilde{\omega} \delta$ 'ov̉ en $\delta \varepsilon \tilde{\imath}$ o en $\alpha i \delta$ oṽ no podría haber ofrecido una solución textual satisfactoria, sobre todo si tenemos en cuenta que la versión "original" en los códices de Dion conocidos presenta siempre, sin excepción, una $\omega$ al comienzo y luego de la $\delta$ el diptongo $\varepsilon v$.

En la edición de J. C. de Pauw del año 1732 el poema, identificado con el número veinte, se encuentra entre los "Carmina et Fragmenta Alia", sección

editores anacreónticos como opción textual (ver, no obstante, n.8 en este trabajo). Una aclaración: los errores textuales que se advierten en las diversas reproducciones del fragmento de Anacreonte reproducidos en este trabajo se encuentran de tal modo en las ediciones a las que pertenecen.

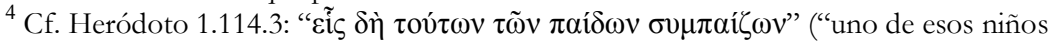
compañeros de juego"), donde el participio del verbo en cuestión no requiere ningún complemento en dativo, significando, por lo tanto, "que jugaban juntos". 
que sigue al corpus poético de los poemas apócrifos. De Pauw mantiene el texto de Estienne sin proponer correcciones a la apertura, pero dice en relación al final: "Scribendum arbitror, $\tau$ òv $\varepsilon \mu$ òv $\delta$ ' (de Pauw, 1732: 271). La propuesta textual del editor holandés podría haber sido apropiada en cuanto a que el verbo $\delta i ́ \delta \omega \mu$ r conjugado en el aoristo imperativo de la segunda persona singular con un complemento verbal en infinitivo constituye ciertamente una fórmula hímnica posible. ${ }^{5}$ Pero al igual que ocurría con las conjeturas de Longepierre (y abstrayéndonos nuevamente de

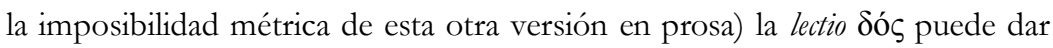
escasa cuenta de su eventual transformación en $\tilde{\omega} \delta^{\prime}$ ov̉ o, más precisamente, en $\tilde{\omega} \delta^{\prime}$ ¿ṽ, según la mayoría de los códices de Dion.

Anterior a la edición de de Pauw es la de Joshua Barnes, que data del año 1705. Se trata de la primera edición en verso de nuestro poema. Aquí el edi-

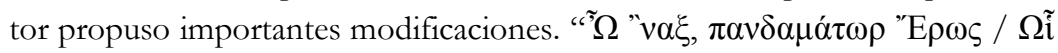
Nú $\mu \varphi \alpha \imath \kappa v \alpha v \omega ́ \pi \imath \delta \varepsilon \varsigma$ " corresponden a los dos primeros versos, mientras que para el último acepta una propuesta textual de Daniel Heinsius: oĩ $\delta^{\prime}, \varepsilon \tilde{v}$ võv

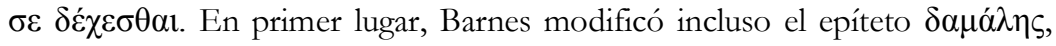
perfectamente legible en los códices. No convencido con la relación establecida por Hesiquio entre el término (que no estaba atestiguado hasta la época de Aristóteles) y el verso anacreóntico, ${ }^{6}$ propone que el epíteto sea

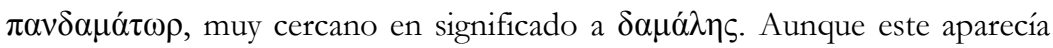
por primera vez aplicado a Eros recién en las Dionisíacas (2. 223) de Nono de Panópolis (s. V d. C.), es probable que Barnes haya justificado su reemplazo basándose en una comparación con el título literario de Eros más conocido, $\lambda v \sigma \mu \varepsilon \lambda \hat{\prime}$ (que afloja los miembros), el cual, al igual que $\pi \alpha v \delta \alpha \mu \alpha ́ \tau \omega \rho$, Homero aplicaba al Sueño. ${ }^{7}$ Independientemente de esto, el epíteto le permitió a Barnes sustituir la superflua interjección $\tilde{\omega}$ por $\pi \alpha v$-, el primer morfema del compuesto adjetival. Por otra parte, atento al régimen del verbo $\sigma v \mu \pi \alpha i \zeta \zeta o v \sigma l$ en el verso cuatro, el editor decidió corregir la conjunción $\kappa \alpha i ́$ con que comienza el segundo verso y aceptar en su lugar $\omega \tilde{i}$, que era una

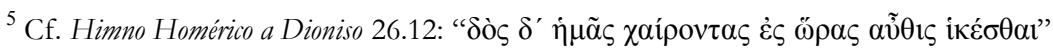
("concédenos arribar con alegría a un nuevo año"). Ver también Liddell \& Scott (1940, s.v. $\delta i \delta \omega \mu \mathrm{i})$.

${ }^{6}$ Exceptuando este fragmento de Anacreonte, la primera aparición del término

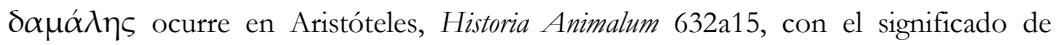
"becerro". En la lírica arcaica se encuentra su correspondiente femenino, $\delta \alpha ́ \mu \alpha \lambda 1 \varsigma$ (Baquílides 19.24). A pesar de que Gentili (1958: 142; 1996: 224), siguiendo a Wilamowitz, Bethe, Fränkel y Lasserre, prefiere conservar para el epíteto de Eros el significado atestiguado en estos usos, ha recibido mayor aceptación la definición que hiciera Hesiquio.

${ }^{7}$ El Sueño es $\pi \alpha v \delta \alpha \mu \alpha ́ \tau \omega \rho$ en Ilíada 24.5 y en Odisea 9.373 , y $\lambda v \sigma \mu \mu \varepsilon \lambda \dot{\varsigma}$ en Odisea 20.57. Este último epíteto acompañó el nombre de Eros en Hesído (Teogonía 120) y Safo (fr. 130 L-P), mientras que Arquíloco (fr. 196 West) lo aplica a róӨos. En el contexto de la lírica arcaica, $\pi \alpha v \delta \alpha \mu \alpha ́ \tau \omega \rho$ fue aplicado al Tiempo (Baquílides 13.205 y Simónides fr. 26.5 PMG). 
conjetura de Isaac Casaubon, con lo que el texto quedaba del siguiente modo: "Oh, señor Eros, que a todos dominas, con quien las Ninfas de oscuros ojos y la purpúrea Afrodita juegan...”. Con estas correcciones podemos suponer que Barnes tenía una intuición en cuanto a la necesidad de introducir en algún lugar de la invocación una cláusula relativa, sólo que, convencido del protagonismo de Eros en la plegaria, debió corregir donde el texto no presentaba problema alguno, demorando el pronombre relativo en caso dativo al segundo verso y no al primero, donde hoy lo leemos.

Por último, para el verso final Barnes acepta una conjetura de Daniel Heinsius: "At optimam omnium Lectionem ad oram Codicis fui Dan. Heinsius

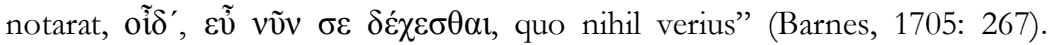
Además del mérito de respetar el esquema del gliconio (pues se trata de un ferecracio, i.e. un gliconio cataléctico), su versión se acerca mucho más al texto de los códices, sobre todo al Urbinas, ya que se corrige solamente la $\omega$ de la fuente. En lo que respecta a la restitución del sentido, en cambio, seguimos bastante lejos del original: "(mi amor) él conoce, ahora tú apruébalo con buen ánimo". En este sentido, debemos mencionar la edición de Boissonade (1823), quien en su de todos modos errónea interpretación se diferencia de otros editores en un punto importante. El editor francés corrige en el último verso la $\omega$ por o, cambia el adverbio eṽ por el de negación ov̉ y desecha el pronombre $\sigma \varepsilon$, colocando en su lugar el verbo $\theta \dot{\lambda} \lambda \varepsilon$. Con estas correcciones el verso es reproducido del siguiente modo: ő $\delta$ 'oṽ vṽv $\theta \dot{\varepsilon} \lambda \varepsilon 1$ $\delta \varepsilon ́ \chi \varepsilon \sigma \theta \alpha$, "(pues mi amor) él (sc. Cleóbulo) no quiere aceptar". Aunque su correcta intuición lo obligó a alterar demasiado el texto, Boissonade tiene el mérito de haber sido el primero en comprender que el sujeto del verbo final debía ser Cleóbulo y no el dios invocado.

La última edición de Anacreonte antes de la corrección de Emperius pertenece a Friedrich Mehlhorn (1825). En este caso los dos primeros versos

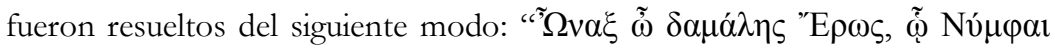
$\kappa v \alpha \nu \omega ́ \pi \imath \varepsilon \varepsilon \varsigma^{\prime}$, con lo que el texto de Mehlhorn resulta similar al de Barnes, aunque evitando la innecesaria transformación del epíteto que hiciera este último. Pero más interesante que su decisión textual considero su nota al primer verso, no tanto por lo que efectivamente dice, sino por el énfasis puesto en la identidad del dios:

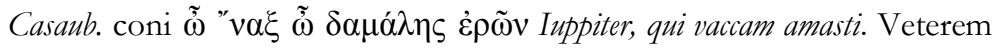

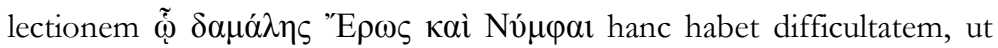
nesciamus, quem Deorum hic allocutus sit Anacreon. (Mehlhorn, 1825: 231)

Habiendo aceptado el pronombre relativo que Casaubon proponía para el segundo verso, Mehlhorn rechaza, por otra parte, la conjetura del francés en el primer verso, mediante la cual había podido proponer que el dios invo- 
cado no era Eros, sino Zeus. Más allá de la intrascendencia de la lectura de Casaubon, jamás considerada como posibilidad en las ediciones de Anacreonte, resulta importante saber que hubo alguna propuesta alternativa que cuestionaba la lectio establecida, por la que Eros resultaba el innegable destinatario de la plegaria. A la reproducción de la conjetura de Casaubon, Mehlhorn agrega además que la lección de Estienne (la vetus lectio) presentaba la dificultad de no poder explicar quién era el dios interlocutor de Anacreonte. ${ }^{8}$ El editor alemán parece no dudar de que se trate del pequeño hijo de Afrodita. $\mathrm{Y}$, sin embargo, su propia nota, a través de la referencia a algunas de las dificultades habidas en torno a la identidad del dios invocado, sincera la inestabilidad textual del primer verso, lo que no acuerda, empero, con la convicción de los filólogos de entonces acerca del protagonismo de Eros.

No obstante esta obstinación, no tuvo que pasar mucho tiempo para que otro filólogo alemán, esta vez un editor de Dion y no de Anacreonte, Adolphus Emperius, resolviera el primer verso a partir de la detección del nombre de Dioniso en la línea final.

\section{La epifanía simposíaca de Dioniso: plegaria, juegos y enigmas literarios}

Los avatares en la historia de la recepción del fragmento de Anacreonte son interesantes por sí mismos, pues ilustran la tarea del editor y del crítico del texto, como nadie advertidos sobre el carácter proteico, a veces hasta ilusorio, de su objeto. Pero independientemente de esto, en el caso concreto del texto que nos ocupa, el anecdotario podría estar señalándonos algo que podría no ser tan exterior al poema mismo. Es muy posible que la corrección de Emperius, reveladora de un texto diferente, haya significado para varios lectores una verdadera sorpresa. Pero ¿podemos adjudicar este efecto sospechado simplemente a la detección del error filológico? ¿No podría ser, acaso, que los varios siglos de lecturas desatinadas no hicieran más que exagerar un resultado buscado y planificado desde un principio? Intuiciones tales nos llevan a desarrollar una hipótesis por la que planteamos que el poema de Anacreonte constituiría una especie de enigma literario en donde la solución no sería otra que el nombre de Dioniso.

Por empezar, diremos que la plegaria no pretende validez cultual y que, por el contrario, comporta un carácter lúdico que suele caracterizar a la canción simposíaca. De acuerdo con esto, Anacreonte utilizaría la estructura de la plegaria religiosa para construir un poema que no habría de tener otro

${ }^{8}$ Sobre esta lección de Estienne ver n. 3 en este trabajo. Cabe la aclaración que también el texto que ofrecía Boissonade presentaba el pronombre relativo en dativo en vez de la interjección, sólo que el editor francés alegaba haberse basado en el códice parisino (B) de Dion (a pesar de Page y de Bergk, quienes leen en todos los códices la interjección). Boissonade tampoco podía explicar quién era el dios interlocutor de Anacreonte. 
contexto de performance más que el del simposio (Del Grande, 1959: 203; Furley \& Bremer, 2001: 177, vol. I; Gentili, 1996: 223). Su calidad poética puede ser juzgada en gran medida por el equilibrio entre el respeto a la forma (de manera tal que el texto pueda, de hecho, ser identificado como una plegaria) y las innovaciones necesarias para que se ajuste al contexto de regocijo dentro de la ocasión mencionada. Entre los elementos que permiten identificar al poema como un bymnos kletikos encontramos, además de la propia apa-

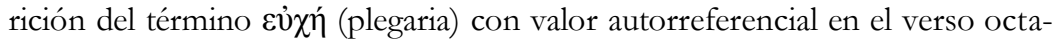
vo, la invocación inicial al dios (aunque no sepamos todavía de quién se trata), su caracterización a partir de actividades y compañías divinas predilectas, la utilización de un léxico habitual en la himnodia, como los verbos

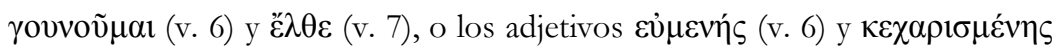
(v. 7), y finalmente la presencia de un pedido concreto a la divinidad invocada. ${ }^{9}$ Por otra parte, y fortaleciendo la pertinencia del poema dentro del contexto simposíaco, debemos destacar el componente sexual implícito en los epítetos de los dioses, así como en el verbo $\sigma 0 \mu \pi \alpha i \zeta \varepsilon ı v ;{ }^{10}$ Patricia Rosenme-

9 Sobre la estructura formal del hymnos kletikos, ver Furley \& Bremer (2001: 52 ss., vol. I) y Race (1982). Los primeros ofrecen una breve descripción de los elementos hímnicos utilizados por Anacreonte en su plegaria (Id., 177-178).

${ }^{10}$ La crítica es unánime en cuanto al sentido sexual de $\pi \alpha$ á̧દev aquí, por cierto documentado con este sentido en otros textos. En lo que respecta a los epítetos, $\delta \alpha \mu \alpha \dot{\lambda} \eta \varsigma$ ha sido explicado por quienes adoptan la definición de Hesiquio (ver n. 6 en este trabajo) a partir de una concepción del amor como fuerza impuesta sobre el amante, que por ello sufre. Incluso quienes se han inclinado por la definición de "becerro" atribuyen al epíteto un sentido erótico, siendo el toro un animal que encarna, según los griegos, la potencia sexual. Al epíteto de las ninfas ( $\kappa \nu \alpha \nu \omega ́ \pi l \delta \varepsilon \varsigma)$ podría subyacer, quizás, un sentido sexual, ya que los ojos oscuros parecen haber sido percibidos de una belleza seductoramente inquietante, incluso inhibidora a juzgar por el empleo

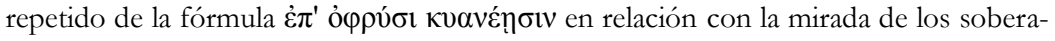
nos olímpicos (Breitenberger, 2007: 186-187). No obstante esta posibilidad, debe decirse que en oportunidades en que el adjetivo acompaña el nombramiento de ciertas deidades femeninas (Anfítrite es kyanopis en Odisea 12.60 y en IG 14.1942 el adjetivo se atribuye a la Musa), no parece implicar necesariamente un sentido sexual. Finalmente, el sentido de $\pi о \rho \varphi \nu \rho \tilde{~}$ aplicado a Afrodita es muy debatido. Se puede enfatizar el sentido cromático (ver ref. a Eliano en n. 2 de este trabajo) o el sentido de movimien-

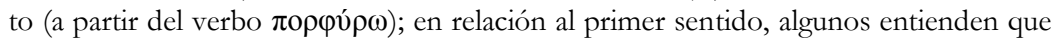
hace referencia al rubor de la piel (siempre un índice de la belleza femenina, cfr. Simónides de Ceos, fr. 585 PMG), otros a los atavíos lujosos con que se solía representar a los dioses (cf. Safo fr. 54 L-P, donde una porphyria kblamys habría vestido a Eros; ver también, Safo fr. 92 L-P e Himno Homérico a Dioniso 7.6). Algunas otras propuestas aúnan la idea de movimiento con el sentido cromático: los objetos así descritos habrían tenido, entonces, una apariencia iridiscente, por lo que traducciones posibles serían "radiante" o "brillante". Por su parte, Goldhill (1987: 13) ofrece un testimonio del uso del adjetivo $\pi$ орфú $\rho \varepsilon о \varsigma$ para indicar el rubor de la piel, pero no como encomio de la belleza de unas mejillas jóvenes, sino referido a un rubor causado por los efectos del amor y del vino. Es posible, no obstante todas estas explicaciones, que más de un sentido estén implicados. Desde nuestro punto de vista, el poema es intencionalmente ambiguo, y el matiz sexual del adjetivo se detectaría una vez terminada la lectura, pues al comienzo el poema se presenta como una simple plegaria y en 
yer (2004: 164-166), por ejemplo, ha ofrecido un breve pero firme análisis de este poema, de acuerdo con el cual a la tríada Dioniso-Eros-Afrodita se la representa en un juego divino (evocando, quizás, el juego conjunto de Hermes, Ares y Apolo en el Himno Homérico a Apolo 3.200-206) que se corresponde con el imperativo del disfrute en el simposio. El juego comunitario de los dioses se replicaría en el ámbito simposíaco, sólo que en su forma más mundana, más terrenal, siendo el juego en este caso de naturaleza principalmente erótica. La invocación a Dioniso debería entenderse, entonces, como una invitación del poeta al dios para que abandone los inocentes recreos divinos y se divierta, en cambio, con Cleóbulo, de quien deberá ser un buen symboulos

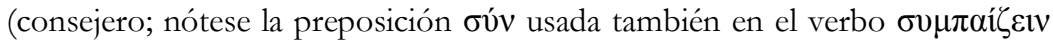
en el verso cuarto), término utilizado algo irónicamente, ya que el verdadero beneficiario de ese equipo sería en definitiva el propio Anacreonte.

Pero a los fines de fundamentar la hipótesis de este trabajo, de entre todas las "anomalías" que presenta esta plegaria nos interesa destacar el retardo extremado en el nombramiento del dios al que se invoca. Se trata de una clara violación a una ley fundamental de la himnodia, según la cual el correcto nombramiento del dios es esencial desde un comienzo, pues este debe saber que es a él y no a otro a quien se está llamando (Furley \& Bremer, 2001: 54, vol. I; Goldhill, 1987: 12). En este sentido, desde un punto de vista pragmático-cultual, la plegaria se muestra claramente inoperante. Pero el contexto simposíaco tampoco parece por sí mismo poder explicar una innovación formal tal. ¿Cuál podría ser, entonces, su fundamento? De acuerdo con nuestra lectura, este "hipérbaton" de la estructura hímnica plantea un interrogante inicial que desafía las expectativas del auditorio del poema y transforma a los oyentes en alocutarios de una especie de acertijo. La formulación de esta invocación imposible tendría así un efecto semejante al de un encantamiento, un hechizo. Como la Esfinge, Anacreonte habría buscado atrapar a su público no solamente mediante la belleza de la palabra mélica, sino, más concretamente, valiéndose de una forma mucho más básica, por su esencia dialógica, de interpelar y comprometer al otro: mediante una pregunta, aquí implícita en la elipsis del nombre del dios que habrá de revelarse. Destaquemos que el término griphos (acertijo) significa en primer lugar "red de pesca", lo que si, por un lado, estaría indicando cierta estrategia poética, por el otro, refiriendo ahora al contenido del poema, revelaría las verdaderas intenciones del yo enamorado: atrapar al hasta el momento esquivo Cleóbulo. Podríamos decir, por tanto, que Anacreonte extiende una misma red que opera en dos niveles: en su identificación con el yo, su plegaria a Dioniso constituye una textura piadosa que actúa como trampa para cumplir con un propósito más bien

este nivel el epíteto exige ser comprendido dentro de las fórmulas tradicionales de la

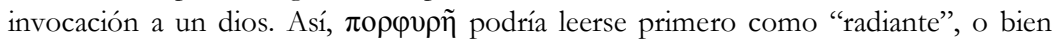
"de piel ruborizada", o bien "ataviada de púrpuras", todas las cuales serían calificaciones admitidas en la apelación a un dios, mientras que el sentido sexual implícito se dejaría entrever cuando se advierte hacia el final el verdadero tono de la invocación. 
profano; como poeta, en-canta a su auditorio proponiendo un poemaenigma.

Quizás sea necesario aclarar que desde un punto de vista puramente formal no estamos ante un acertijo. ${ }^{11}$ Sin embargo, no podemos no reconocer que el poema presenta la ambigüedad definitoria de la formulación enigmática: la elipsis de un concepto que habrá finalmente de revelarse, acompañada de algunas claves que apuntan a dicho concepto. ${ }^{12}$ En este sentido, debemos tener en cuenta la descripción del dios, pero también los indicios en el mismo pedido; Anacreonte le pide a Dioniso que sea "un buen

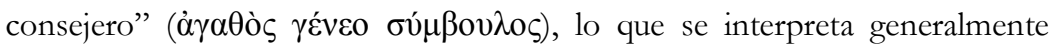
como un juego de palabras con el nombre de Cleóbulo (K $\lambda \varepsilon$ ó $\beta o v \lambda o \varsigma)$, pero que podría estar remitiendo al mismo tiempo a un epíteto que suele acompañar la mención de Dioniso: عủßov $\lambda \varepsilon u ́ \varsigma$ ("quien ofrece consejos valiosos").

En cuanto al (probable) contexto de performance del poema que nos ocupa, debemos recordar que las adivinanzas habrían constituido un juego habitual de los symposia. Esto nos lo relata Ateneo (10.448b-453b), una de las principales fuentes de griphoi griegos. ${ }^{13}$ Entre los griphoi allí referidos algunos poseen un indudable carácter literario, como la (hoy desconocida) oda asigmática de Píndaro. ${ }^{14}$ Recordemos, además, los varios enigmas presentes en la colección de elegías atribuidas a Teognis (vv. 257-60; 261-66; 1209-15; 1229-30). Por otra parte, determinados pasajes o fragmentos de poetas arcaicos presentaban a los eruditos alejandrinos ciertos desafíos de índole lexical, razón por la que los trataban como problemas (problemata) cuyas respectivas soluciones (lyseis) dependían de un proceso de investigación o búsqueda (zetesis). En este sentido, los pasajes poéticos constituían verdaderos enigmas filológicos. Uno de estos fragmentos pertenece a Alceo (fr. 359 en la edición

\footnotetext{
${ }^{11}$ Nos referimos principalmente a la enunciación típica del acertijo como pregunta, a lo que podemos sumar su particular formulación a partir de la unión de dos imágenes o conceptos que desde una lógica cotidiana no podrían corresponderse. Para una descripción del acertijo como forma tradicional ver un repaso en Taylor (1943), el análisis de Pepicello \& Green (1984) y los diferentes ensayos recogidos en HasanRokem \& Shulman (1996).

${ }^{12}$ Ver Pagis (1996: 81): "Every proper riddle must fulfill two conditions: the first is its social function as a competition between the riddler and the riddlees; the second is its literary form, which must be difficult and enigmatic, yet containing the clues needed to decipher it. (...) A riddle is only that text which is intended to function as a riddlea text whose author, or later riddler, deliberately presents it to the reader as a challenge". Para profundizar acerca del lenguaje oscuro, enigmático, y la poesía puede consultarse Tiffany (2009).

${ }^{13}$ Focio s. v. $\gamma \rho \tilde{i} \varphi o t$ llega a definir los acertijos como symposiaka zetemata. Mucho antes, Platón (República 479b-c) ya se refería a ciertos juegos basados en el doble sentido de las palabras como diversiones típicas de los banquetes.

${ }^{14}$ Ateneo no transcribe el texto pindárico al que refiere, pero Dioniso de Halicarnaso (De compositione verborum 14) cita un ditirambo de Píndaro (fr. 70b) como ejemplo de asigmatismo. Aquí, sin embargo, no se trata de una composición que prescinde del sonido de la sigma (pues, de hecho, sí hay sigmas), sino de una referencia al sonido

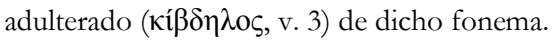


de Lobel \& Page, 1997), transmitido a nosotros por vía de Ateneo (3.85f) en el contexto de una discusión acerca de los nombres de diversos peces y moluscos:

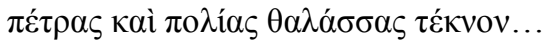

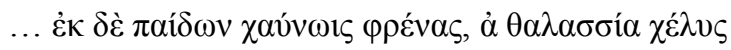

Vástago de la piedra y del canoso mar...

... relajas la mente de los jóvenes, tú, tortuga marina

El problema radicaba en si la palabra empleada por Alceo en el verso final era $\lambda \varepsilon \pi \alpha ́ s$ (lapa de mar), como lo transmite Ateneo citando a Calias de Mitilene y aludiendo a la explicación de Dicaerco, o $\chi \varepsilon ́ \lambda u \varsigma$ (tortuga marina), como prefería Aristófanes de Bizancio (y muchos de los editores modernos). El fragmento continúa siendo hoy en día discutido, pero dicha discusión no se basa tanto en si es Aristófanes más creible como filólogo que Calias, sino en una interpretación que debemos a Wilamowitz (cit. West, 1990: 6), aceptada desde entonces: que el poema alcaico habría sido originalmente un acertijo. Así, podemos, como Slater (1982: 338-339), suscribir a la propuesta de Aristófanes y aceptar que el término final sea $\chi \varepsilon ́ \lambda v \varsigma$, sólo que debe ser interpretado metafóricamente como "lira", el instrumento hecho con el caparazón de la tortuga. O podemos acordar con West (1990: 6) en que Dicaerco y Calias tenían en su texto la solución del acertijo, es decir, que la palabra $\chi \varepsilon ́ \lambda \nu \varsigma$ se encontraba en el poema original, pero que la "tortuga marina" refiere, en realidad, a la $\lambda \varepsilon \pi \alpha ́ \varsigma$, un molusco que lleva caparazón.

De esta alusión al fragmento de Alceo nos interesa destacar dos cuestiones. Primero, que de manera similar a lo que hemos propuesto aquí como hipótesis acerca de las dificultades para interpretar el texto de Anacreonte en su aspecto material, el acertijo alcaico constituyó asimismo un enigma filológico para sus exegetas, ya en la misma Antigüedad. Puede tratarse de una simple coincidencia, pero tampoco sería tan ilógica una relación de causalidad entre el enigma conceptual y el enigma textual al que se enfrenta el filólogo. El ocultamiento/revelación del término-respuesta, posibilitado por la ambigüedad intencionada de su enunciación, puede, sobre todo en un contexto cultural de transmisión escrita endeble, desembocar en un texto enigmático en su misma formulación (como el caso del fragmento de Alceo) y que, de manera extrema, puede también comprometer su integridad material (como el caso del poema de Anacreonte en la modernidad). En segundo lugar (aunque solamente si seguimos a Slater), podemos presentar el acertijo de Alceo como un antecedente de la plegaria enigmática de Anacreonte en la medida en que aquel, al igual que este, daría a conocer la solución al final del mismo poema. Es cierto que en el caso de Alceo la respuesta se revela de forma indirecta, pues no se trata literalmente de la tortuga, sino de la lira construida 
con su caparazón. Pero seguramente el auditorio habría reconocido de inmediato y con regocijo la alusión mitológica a la creación del instrumento por parte del dios Hermes. Por su lado, el deleite en la resolución del poema de Anacreonte tendría que ver, según nuestra apreciación de la experiencia filológica, con la aparición final del nombre de Dioniso. Su mención no solamente ofrece la respuesta del enigma, sino que además -y aquí radica lo más interesante que puede aportar nuestra lectura- hace posible la epifanía del dios, exigida en cierta forma por la misma estructura hímnica. Dicho con otras palabras y ya a modo de conclusión: lo que sostenemos es que el formato tradicional del acertijo, diseñado como una pregunta que exige la réplica de un otro, se ve sustituido por el trabajo perito del poeta con la materia himnódica. Al conceptualizar el nombre de Dioniso en términos de una respuesta a una especie de adivinanza planteada como la formulación truncada de la invocación inicial, no podemos más que proponer el carácter de revelación que se le confiere al nombre del dios. Esto, en el marco de la codificación lingüística del universo literario, no puede resultar sino en una verdadera epifanía. Decimos, entonces, que la anulación del valor pragmático de una plegaria que no requiere realmente del arribo efectivo de un dios invocado en un contexto cultual es compensada por Anacreonte con el uso lúdico de la forma hímnica, en tanto el juego literario planteado como enigma requiere, por cierto, de una revelación. Paradójicamente, la epifanía de Dioniso es el logro de esta plegaria de escaso valor religioso.

\section{Observación final}

Habiendo así concluido, permítaseme este espacio epilogal para agregar una hipótesis que no desarrollaré, pero que posiblemente merezca ser enunciada. $\mathrm{Si}$, tal como afirma el consenso crítico, la plegaria se encuentra perfectamente justificada dentro de un contexto simposíaco afecto a los juegos de todo tipo, incluidos los literarios, la epifanía de Dioniso en particular podría no haber quedado reducida a su expresión verbal. Por el contrario, una aparición del dios en el simposio podría muy bien hacerse tangible en el vino que algún joven copero habría de haber acercado al poeta (ver, por ejemplo, Anacreonte fr. 356 PMG). Sin pretender aquí recrear una supuesta situación original, lo que sí podemos decir con certeza es que Dioniso sería un mejor epikouros de Anacreonte en la tarea de convencer a Cleóbulo, si el dios, más que en su aspecto ritual de macho cabrío, se revelase en el banquete con la forma de aquel zumo que él mismo obsequió al hombre para liberarlo de las penas cotidianas. ${ }^{15}$

\footnotetext{
${ }^{15}$ Furley \& Bremer (2001: 177, vol. I) sugieren una escena similar, en la que la enun-
} ciación de la plegaria coincidiría con la imagen de una copa de vino levantada a modo 


\section{Referencias}

1. Ediciones:

BARNES, Josua (1705). Anacreon Teius, poeta lyricus... Cantabrigii: Typis Academicis.

BERGK, Theodorus (1834). Anacreontis Carminum Reliquias. Lipsiae: Sumtu Reichenbachiorum Fratrum.

BOISSONADE, J. F. (1823) ANAKPESN. Anacreontis Reliquiae, Basilii, Iuliani, Pauli Silentiarii Anacreontica. Parisiis: Lefevre.

DEL GRANDE, Carlo (1959). Phorminx. Antologia della lirica greca. 2 da. ed. ampliada. Napoli: Luigi Loffredo.

EMPERIUS, Adolphus (1844). Dionis Chrysostomi Opera Graece. Brunsvigae: G. Westermanni.

GENTILI, Bruno (1958). Anacreonte. Roma: Ateneo

LOBEL, Edgar \& Denys Page (1997). Poetarum Lesbiorum Fragmenta. Special Edition for Sandpiper Books Ltd. Oxford: Oxford Clarendon Press.

LONGEPIERRE, Hilaire-Bernard de (1962). Les Ouvres d'Anacréon et de Sapho... Paris: Charles Clouzier.

MEHLHORN, Fridericus (1825). Anacreontea quae dicuntur... Glogaviae: Libraria Nova Guenteriana.

MICHELANGELI, Luigi Alessandro (1882). Anacreonte. Edizione critica. Bologna: Nicola Zanichelli.

PAGE, Denys (1962). Poetae Melici Graeci. Oxford: Oxford University Press.

DE PAUW, Joannes Cornelius (1732). Anacreontis Teii Odae et fragmenta. Trajecti ad Rhenum: Guilielmus Kroon.

STEPHANUS, Henricus (1566). Carminum Poetarum novem lyricae poesecs principum, fragmenta. 2da. ed. Lutetiae: Huldrichi Fuggeri typographus.

(1560). Carmina Poetarum novem lyricae poesews principum, fragmenta. Lutetiae: Huldrichi Fuggeri typographus.

2. Textos críticos y obras de referencia:

BREITENBERGER, Barbara (2007). Aphrodite and Eros. The Development of Erotic Mythology in Early Greek Poetry and Cult. New York-Oxon: Routledge.

EMPERIUS, Adolphus (1830). Observationes in Dionem Chrysostomum. Lipsiae: F.C.G. Vogel.

FURLEY, William D. \& Jan Maarten Bremer (2001). Greek Hymns. Selected Cult Songs from the Archaic to the Hellenistic Period, vols. 1 y 2. Tübingen: Mohr Siebeck.

GENTILI, Bruno (1996). Poesía y público en la Grecia Antigua. Trad. Xavier Riu. Barcelona: Quaderns Crema.

GOLDHILL, Simon (1987). "The Dance of the Veils: Reading Five Fragments of Anacreon." Eranos 85: 9-18.

de brindis. Propongo pensar, además, en ese efecto de "revelación" del cual he intentado dar cuenta en este artículo. 
HASAN-ROKEM, Galit \& David Shulman, eds. (1996). Untying the Knot. On Riddles and Other Enigmatic Modes. New York-Oxford: Oxford University Press.

LIDDELL, H.G. \& R. Scott (1940). Greek-English Lexicon. 9na. ed. Oxford: Oxford Clarendon Press.

PAGIS, Dan (1996). "Toward a Theory of the Literary Riddle." Untying the Knot. On Riddles and Other Enigmatic Modes. Eds. Galit Hasan-Rokem y David Shulman. New York-Oxford: Oxford University Press. 81-108.

PEPICELLO, W. J. \& Thomas A. Green (1984). The Language of Riddles. New Perspectives. Columbus: Ohio State University Press.

RACE, William H. (1982). "Aspects of Rhetoric and Form in Greek Hymns." Greek, Roman \& Byzantine Studies 23.1: 5-14.

ROSENMEYER, Patricia (2004). "Girls at Play in Early Greek Poetry." American Journal of Pbilology 125: 163-178.

SLATER, William J. (1982). "Aristophanes of Byzantium and ProblemSolving in the Museum." The Classical Quarterly 32.2: 336-349.

TAYLOR, Archer (1943). “The Riddle.” California Folklore Quarterly 2.2: 129147.

TIFFANY, Daniel (2009). Infidel Poetics. Riddles, Nightlife, Substance. Chicago: The University of Chicago Press.

WEST, Martin L. (1990). "Notes on Sappho and Alcaeus." Zeitschrift für Papyrologie und Epigraphik 80: 1-8. 